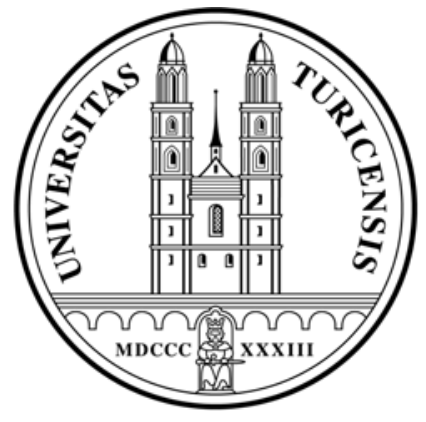

Institute for Empirical Research in Economics

University of Zurich

Working Paper Series

ISSN 1424-0459

Working Paper No. 412

\title{
Uncertainty Breeds Decreasing Impatience: The Role of Risk Preferences in Time Discounting
}

Thomas Epper, Helga Fehr-Duda and Adrian Bruhin

May 2009 


\title{
Uncertainty Breeds Decreasing Impatience: The Role of Risk Preferences in Time Discounting
}

\author{
Thomas Epper* Helga Fehr-Duda Adrian Bruhin
}

May 18, 2009

\begin{abstract}
Future events are uncertain by their very nature. Therefore, people's risk preferences are likely to play a role in the valuation of allegedly guaranteed future outcomes. We show that future uncertainty conjointly with people's proneness to nonlinear probability weighting generates a unifying framework for explaining many anomalies in intertemporal choice, such as hyperbolic discounting and subadditivity of discount factors. Moreover, our approach implies that higher uncertainty of future prospects increases the hyperbolicity of discount rates, suggesting that institutional deficiencies such as lack of contract enforcement, may be a source of hyperbolic discounting behavior. Based on an experiment with monetary incentives, we show that people's risk taking behavior is indeed a significant determinant of their time discounting behavior: Greater departures from linear probability weighting predict a stronger decline in impatience on the level of individual behavior.
\end{abstract}

Keywords: Institutionally Generated Uncertainty, Time Preferences, Risk Preferences, Hyperbolic Discounting, Probability Weighting

JEL: D01, D81, D91

${ }^{*}$ Corresponding Author: Thomas Epper, ETH Zurich, Chair of Economics, Weinbergstrasse 35, CH8092 Zurich, Switzerland; epper[at]econ.gess.ethz.ch. Acknowledgements: We thank Ernst Fehr, Urs Fischbacher, Drew Fudenberg, David Laibson, Andrew Oswald, Drazen Prelec, and Herbert Walther for helpful comments. The usual disclaimer applies. This research was supported by the Swiss National Science Foundation (Grant 100012-109907). 
"Future income is always subject to some uncertainty, and this uncertainty must naturally have an influence on the rate of time preference, or degree of impatience, of its possessor."

Fisher (1930)

\section{Introduction}

Every day of our lives we take decisions, some with immediate consequences, some others with consequences in the distant future. While immediate consequences, depending on the nature of the decision, may be certain or uncertain, delayed ones are bound to be uncertain even when they are allegedly guaranteed: For instance, a promised reward may, due to unforeseen circumstances, materialize later or turn out to be smaller than expected, or sudden illness or death may keep the decision maker from collecting her reward. For these reasons, future consequences are inextricably associated with uncertainty, implying that the decision maker's valuation of temporal prospects not only depends on her pure time preference, i.e. her preference for immediate utility over delayed utility, but also on her perception of uncertainty and, consequently, on her risk preferences. In other words, uncertainty drives a wedge between pure time preferences and time discounting.

If this account is an accurate description of intertemporal choice it has far reaching implications for observed discounting behavior, the most obvious one being that behaviorally revealed discount rates will be higher than the rate of pure time preference as they include a risk premium. Not surprisingly then, uncertainty has been identified to be an important confound in the measurement of time preferences, which may, at least partly, explain the notoriously high discount rates found in empirical studies (Frederick, Loewenstein, and O'Donoghue, 2002). Recent research has not focused on the magnitude of observed discount rates, however, but rather on their hyperbolicity: Frequently, people's preference for present consumption seems to be so strong that their impatience declines rapidly when the good in question is not available immediately, but at some more remote future time (Thaler, 1981; Benzion, Rapoport, and Yagil, 1989; Ainslie, 1992; Chapman and Elstein, 1995; Chapman, 1996). ${ }^{1}$ Apparently, such a behavior is not consistent with exponential discounting.

\footnotetext{
${ }^{1}$ Consequently, a large number of models of decreasing impatience, commonly labeled "hyperbolic" or "quasi-hyperbolic" discounting, have been developed and applied to such diverse fields as saving behavior, procrastination, addiction, retirement decisions, and global warming (Loewenstein and Prelec, 1992; Laibson, 1997, 1998; Laibson, Repetto, Tobacman, Hall, Gale, and Akerlof, 1998; O'Donoghue and Rabin, 1999; Richards, Zhang, Mitchell, and Wit, 1999; Angeletos, Laibson, Repetto, and Tobacman, 2001; O'Donoghue and Rabin, 2001; Karp, 2005).
} 
In this paper we show, and provide experimental support for our claim, that uncertainty affects not only the level of discount rates but also their decline if people's risk preferences are adequately accounted for. We derive our results by referring to a frequently observed characteristic of risk taking behavior: Often people's preference for a smaller more probable outcome over a larger less probable one changes in favor of the larger outcome when both outcome probabilities are scaled down by a common factor (Starmer and Sugden, 1989). A prominent example of this regularity is the Allais paradox. Such behavior can be suitably captured by decision models that incorporate a nonlinear probability weighting function exhibiting subproportionality. Subproportionality means that for a fixed ratio of probabilities the ratio of the corresponding probability weights is closer to unity when the probabilities are low than when they are high (Kahneman and Tversky, 1979). Intuitively speaking, scaling down the original probabilities makes them less distinguishable from each other and, therefore, the larger outcome tends to become more salient to the decision maker. That nonlinear probability weighting is the rule and not the exception has recently been shown by a finite mixture regression study which classifies individual decision makers according to their observed risk taking behavior: Bruhin, Fehr-Duda, and Epper (2008) find that, in three different data sets, 80\% of the subjects exhibit substantial departures from linear probability weighting, whereas only $20 \%$ are not prone to such behavior.

We show that subproportionality in conjunction with intrinsic uncertainty directly generates hyperbolicity of discounting behavior. Furthermore, we link the extent of decreasing impatience to the degree of subproportionality: The more substantially a decision maker's probability weighting curve departs from linearity, the more strongly will impatience decline over time, ceteris paribus, i.e. the more pronouncedly nonexponential will be her discounting pattern.

The crucial question then remains whether probability weighting indeed predicts time discounting behavior. That risk taking and discounting behavior exhibit a number of parallels has already been noted by Prelec and Loewenstein (1991), but so far no empirical evidence has been presented that a link between probability weighting and time discounting actually exists on the level of individual behavior, a sine qua non for our model.

In order to supply empirical support for our hypothesis we conducted an experiment with salient real monetary incentives, which exhibits a number of distinguishing features: First, we generated data rich enough to be able to estimate individual probability weighting functions and relate them to the same subjects' discount rates. Second, since the essential assumption of our approach is that decision makers perceive future outcomes as uncertain even when these are allegedly guaranteed, a stringent test of the theory re- 
quires that promised rewards indeed appear guaranteed. For that purpose, in contrast to many previous discounting experiments, every single subject was paid in an incentive compatible manner. Furthermore, subjects received official vouchers of the Swiss Federal Institute of Technology, an institution with an impeccable reputation, which entitled them to collect their delayed payoffs at the university cashier's desk. Third, we kept transaction costs equal across different payment dates in order to preclude confounding effects. Finally, we controlled for concave outcome utility.

We present the following results. First, we show that the degree of subjects' subproportionality of probability weights is a highly significant determinant of the strength of decreasing impatience, as predicted by our framework. The curvature of the utility function, however, seems not to be directly related to the decline of impatience. Second, estimation results are robust to controlling for socioeconomic characteristics, such as gender, age, experience with investment decisions and cognitive abilities. In fact, the only variable predicting decreasing impatience turns out to be the degree of nonlinearity of probability weights, which explains a, by any standard, large percentage of the variation in the extent of the decline. Moreover, all our results are insensitive to model specification.

Furthermore, we show that, aside from hyperbolic discounting, the uncertainty-cumprobability-weighting approach provides a unifying framework for explaining a number of other puzzling empirical findings as well. First, losses have been consistently found to get discounted less heavily than gains, usually referred to as gain-loss asymmetry or sign effect. Second, there is evidence of diminishing immediacy, i.e. people's preference for present outcomes weakens drastically when the present becomes risky: Whereas the majority of subjects prefer a smaller immediate reward to a larger delayed one, merely a minority do so when both rewards materialize just with a certain probability (Keren and Roelofsma, 1995). Third, risk tolerance seems to be delay dependent as people are more risk tolerant for lotteries played out in the more remote future than for otherwise identical lotteries resolved in the more immediate future (Noussair and $\mathrm{Wu}, 2006$ ). Fourth, Read (2001) presents evidence that observed discounting behavior is subadditive, meaning that discounting over a delay is greater when the delay is divided into subintervals than when it is left undivided. Finally, our model provides a theoretical underpinning of empirical evidence that subjective probabilities attached to temporal prospects decline hyperbolically with the delay of the prospect (Takahashi, Ikeda, Hasegawa, and Greene, 2007).

Our experimental findings strongly support the hypothesis that intrinsic uncertainty is an important factor in generating hyperbolic discounting patterns, even if pure time preferences are exponential. In addition, we show theoretically that an increase in the un- 
certainty of future prospects will increase the hyperbolicity of discount rates. This feature of our approach is important because it implies that hyperbolic discounting is not merely a property of people's risk and time preferences, but may also be affected by institutional characteristics that influence the uncertainty of future prospects. For example, if protection of property rights and institutions of contract enforcement are weak, future prospects are likely to be highly uncertain. In such an environment decision makers that are prone to probability distortions will exhibit a much higher degree of hyperbolic discounting compared to an environment in which the legal system reliably enforces contracts and protects property rights. This link between the institutional sources of uncertainty and discounting behavior may explain why measured discount rates in developing countries, which are often characterized by highly imperfect contract enforcement institutions, exhibit a dramatically more pronounced hyperbolicity than in Western countries (Poulos and Whittington, 2000; Anderson, Dietz, Gordon, and Klawitter, 2004).

Our contribution is related to recent theoretical work by Halevy (2008) who shows that diminishing impatience may be a consequence of nonlinear probability weighting. However, we demonstrate that nonlinear probability weighting in combination with intrinsic uncertainty not only implies hyperbolic discounting but also provides a unifying framework for explaining many other observed anomalies of intertemporal choice. In addition, we present the first evidence that people with more pronounced proneness to probability weighting indeed exhibit more strongly declining impatience. Finally, we show that hyperbolic discounting is not merely a property of individual preferences but may also be generated by the institutional environment.

While uncertainty may be an important channel through which hyperbolicity of discount rates is generated there may be other sources of hyperbolic discounting behavior as well. For instance, pure time preferences may be hyperbolic per se, which is particularly plausible in the context of addictive behavior. And when visceral motives, such as hunger or lust, come into play, uncertainty may not be the dominant dimension decision makers are concerned about. An excessive preference for the present may then be driven by factors other than potential disappearance of the object of desire.

The remainder of the paper is structured as follows: Section 2 illustrates our central hypothesis on the role of risk preferences in time discounting. In Section 3 we describe the experimental design and procedures. Section 4 outlines our approach to estimation. Section 5 presents our results, discussed in detail in Section 6. 


\section{Framework}

In the following section we argue that subproportionality of probability weights is sufficient for hyperbolicity of discounting behavior if the future is perceived as uncertain. We present the formal derivation of this result, which holds for any subproportional probability weighting function, as well as all the other formal proofs in Appendix A. In Section 2.1, we derive comparative static effects using, for simplicity, a specific functional form of the probability weighting curve. Furthermore, we discuss the implications of subproportionality for important empirical regularities in time discounting behavior in Section 2.2 and demonstrate the effects of increasing uncertainty on short-term impatience in Section 2.3 .

In order to derive the presumed link between risk preferences and time discounting we need to specify several ingredients of our approach: First, in order to show that intrinsic uncertainty generates hyperbolicity of discounting behavior, even if pure time preferences are exponential, we assume that pure time preferences are characterized by a constant per-period rate $r \geq 0$. Second, we model intrinsic uncertainty in the following way: Any allegedly guaranteed future payment is perceived to materialize with a constant perperiod probability of contract survival $s, 0<s \leq 1$, which in turn gets transformed into a subjective probability weight by the decision maker's probability weighting function $w$ satisfying $w^{\prime}>0, w(0)=0, w(1)=1$. We assume the probability weighting function to be subproportional. Subproportionality means that for a fixed ratio of probabilities the ratio of the corresponding probability weights is closer to unity when the probabilities are low than when they are high. Expressed formally (Prelec, 1998), subproportionality holds if $1 \geq p>q>0$ and $0<\lambda<1$ implies the inequality

$$
\frac{w(p)}{w(q)}>\frac{w(\lambda p)}{w(\lambda q)} .
$$

According to our framework, a decision maker evaluates a (certain) payment $y_{t}$ delayed by $t$ as a simple risky prospect $\left(y_{t}, s^{t}\right)$ that pays out $y_{t}$ with probability $s^{t}$ and zero otherwise. This implies that the present equivalent $y_{0}$ of the future payment $y_{t}$, such that the decision maker is indifferent between $y_{0}$ and $y_{t}$, is defined by

$$
v\left(y_{0}\right)=v\left(y_{t}\right) \rho^{t} w\left(s^{t}\right),
$$

where $v$ denotes the decision maker's valuation of monetary outcomes $y$ and $\rho$ is defined as $\exp (-r)$. The discount factor $D(t)$ at delay $t$ equals the weight attached to $v\left(y_{t}\right)$, i.e.

$$
D(t)=\rho^{t} w\left(s^{t}\right)
$$


which depends not only on the pure rate of time preference $r$, but also on the probability of contract survival $s$ as well as on the shape of the probability weighting function $w$. Clearly, if $w$ is linear, $D(t)$ declines exponentially irrespective of the magnitude of $s$. If $0<s<1$, the resulting discount factors are lower than for $s=1$, implying that uncertainty per se increases the absolute level of impatience, but cannot account for impatience declining over time. If, however, $w$ is nonlinear and $0<s<1$, the component $w\left(s^{t}\right)$ distorts the discount factor: As we show in Appendix A, subproportionality of $w$ is sufficient for generating hyperbolicity of $w\left(s^{t}\right)$ and, consequently, decreasing impatience if the future is perceived as uncertain.

\subsection{Subproportionality and Hyperbolicity}

For simplicity, we discuss the effects of probability weighting on $D(t)$ with an exemplary illustration. We assume a specific functional form for the probability weighting curve, consistent with the empirical literature, and vary the degree of its nonlinearity.

As we rely on subproportionality as the crucial characteristic of the probability weighting function, we adopt the parameterization suggested by Prelec (1998),

$$
w(p)=\exp \left\{-(-\ln p)^{\alpha}\right\}, 0<\alpha<1 .
$$

This function is subproportional everywhere and, additionally, inverse S-shaped, which is in accordance with abundant empirical evidence (Tversky and Kahneman, 1992; Gonzalez and Wu, 1999; Abdellaoui, 2000; Bruhin, Fehr-Duda, and Epper, 2007). It conveniently captures the degree of departure from linearity by its single parameter $\alpha$ : A smaller value of $\alpha$ implies a more subproportional and more S-shaped probability weighting curve. For values of $\alpha$ within the interval $(0,1)$ the curve intersects the identity line from above at $p=1 / e$, implying overweighting of survival probabilities for $s^{t}<1 / e$ and underweighting for $s^{t}>1 / e$, respectively. Panel $A 1$ of Figure I graphs this specification of the probability weighting function for three distinct parameter values of $\alpha$ : a medium-sized departure from linearity $(\alpha=0.5)$, a strong departure from linearity $(\alpha=0.2)$, as well as the limiting case of linear probability weighting $(\alpha=1) .{ }^{2}$ The dashed curve corresponding to $\alpha=0.2$ is more strongly S-shaped and comparatively more subproportional than the dotted curve for $\alpha=0.5$.

Panel B1 of Figure I depicts, for each of the three cases of probability weighting, the discount factors resulting from Equation 3 as they evolve over time. For a decision maker with linear probability weighting the discount function, represented by the solid

\footnotetext{
${ }^{2}$ For illustrative purposes, in Figure I $r$ is fixed at 0.1 and $s$ is assumed to be 0.8 , which means that $80 \%$ of all contracts are anticipated to survive at least one period.
} 
Figure I: Comparative Statics: Varying $\alpha$ and $s$

Panel A1 - Probability Weighting Function

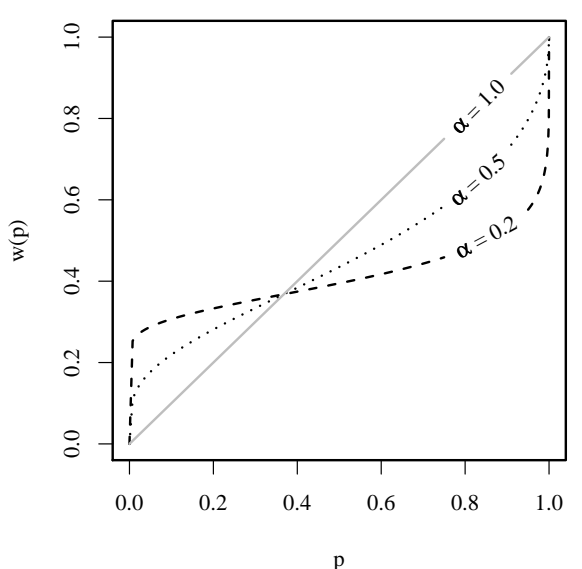

Panel B1 - Discount Function

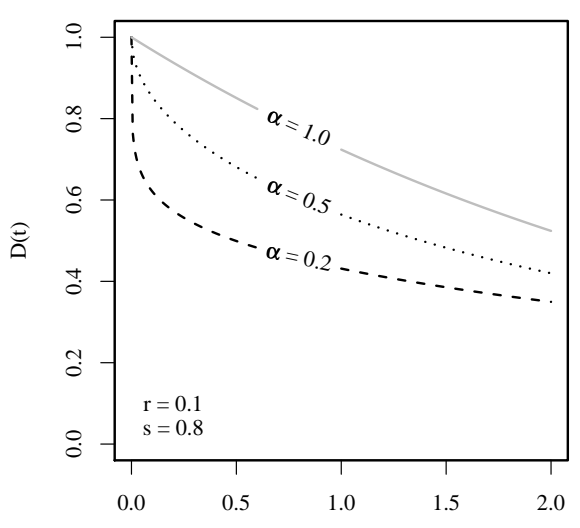

Panel C1 - Imputed Discount Rate

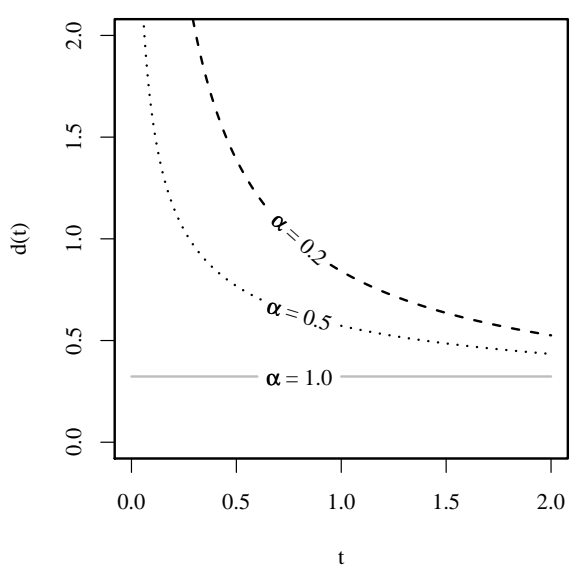

Panel A2 - Probability Weighting Function

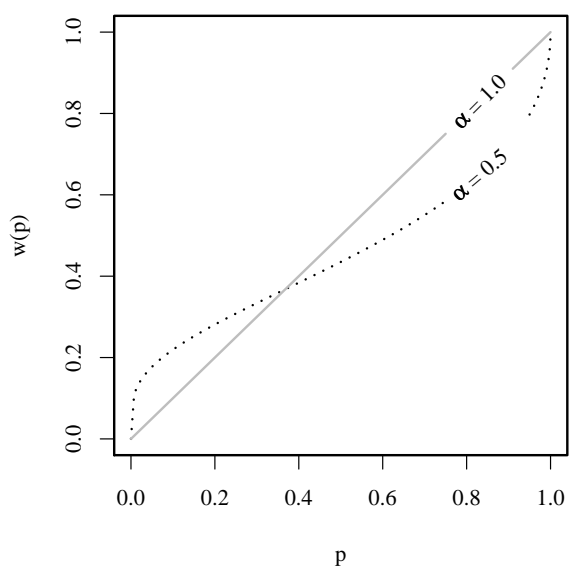

Panel B2 - Discount Function

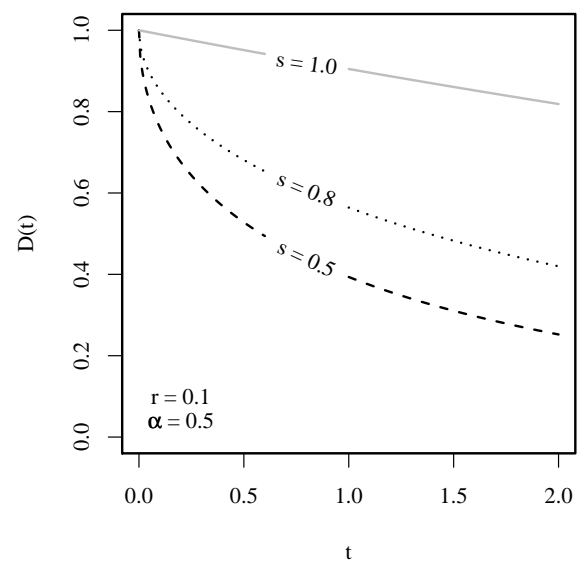

Panel C2 - Imputed Discount Rate

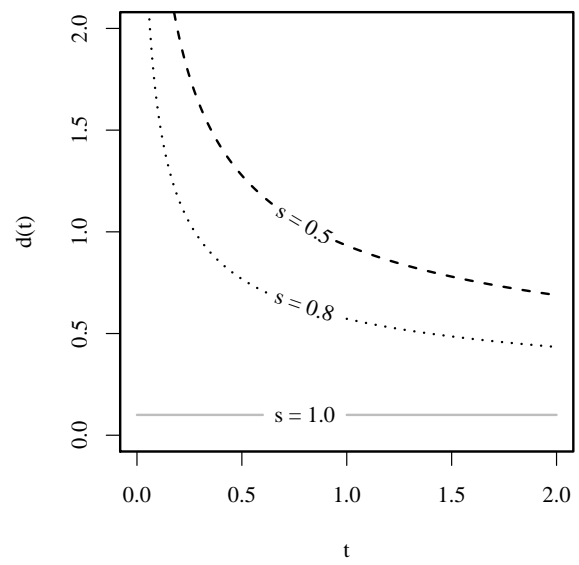


gray curve, is exponential. In contrast, the dotted discount function of a typical decision maker with $\alpha=0.5$ departs from exponentiality, exhibiting an apparently hyperbolic pattern consistent with numerous empirical findings (e.g. Rachlin, Raineri, and Cross (1991); Kirby and Maraković (1995); Myerson and Green (1995); Kirby (1997)). By comparison, the decision maker characterized by the more strongly S-shaped probability weighting curve underweights (overweights) large (small) probabilities more strongly than does the decision maker with $\alpha=0.5$, which leads to an even more pronounced departure from exponential discounting (dashed curve). This relationship constitutes our central hypothesis which we will test empirically.

Finally, Panel $C 1$ of Figure I displays the imputed discount rates $d(t)$ inferred from $D(t)=\exp (-d(t) t)$. The solid gray line corresponds to linear probability weighting. Since this decision maker is not prone to probability distortions, her discount rate is independent of time delay and, consequently, constant over time. In contrast to this decision maker, the discount rates of the decision makers with nonlinear probability weights start out at very high levels and then decline sharply. As is evident from comparing the dashed curve with the dotted one, the more subproportional probability weighting function generates a larger decline in discount rates between period 2 and period 1, i.e. the difference $d(2)-d(1)$ is greater for higher degrees of nonlinearity, measured by $\alpha$, supplying us with an operational hypothesis to be tested.

\subsection{Subproportionality and Anomalies in Time Discounting}

Interpreting future outcomes as inherently uncertain generates not only a link between decreasing impatience and nonlinear probability weighting, but also provides a unifying framework for explaining other qualitative patterns of observed discounting behavior, often termed anomalies as they are, prima vista, in conflict with the assumptions of the standard model of intertemporal choice. Our explanation rests exclusively on subproportionality of probability weighting in combination with intrinsic uncertainty and does not require any further assumptions.

In particular, one robust empirical finding concerns the gain-loss asymmetry in time discounting: Losses have been persistently found to get discounted less heavily than gains when moved into the future (Thaler, 1981; Benzion, Rapoport, and Yagil, 1989; Chapman, 1996). In order to solve this puzzle we resort to the experimental evidence in the domain of risk taking behavior: Estimated probability weighting curves for losses tend to be significantly more elevated than the curves for gains, implying that, for each level of probability, probability weights for losses are larger than for gains (e.g. Abdellaoui (2000)). Consequently, the discount factor $D(t)$ is higher for losses than for gains, as can be inferred from Equation 3, entailing a lower devaluation of losses. Additionally, 
probability weights for losses tend to depart less strongly from linearity (Fehr-Duda, de Gennaro, and Schubert, 2006), implying a comparatively lower degree of hyperbolicity of the ensuing discount function, which seems to be in accord with the empirical evidence reported in Benzion, Rapoport, and Yagil (1989).

Our framework also provides a rationale for evidence of diminishing immediacy when risk comes into play (Keren and Roelofsma, 1995). Keren and Roelofsma find that the percentage of subjects choosing an immediate smaller reward $x$ over a delayed larger reward $y$ decreases significantly when the rewards are not certain but exposed to the same level of probability of materializing, say $p$. Such behavior follows directly from the subproportionality of the probability weighting function. ${ }^{3}$ In both types of decision situations, $(x, p)$ versus $\left(y, p s^{t}\right)$ and $(x, 1)$ versus $\left(y, s^{t}\right)$, the ratios of the subjective probabilities of the delayed and the immediate rewards are the same, namely $1 / s^{t}$. However, the absolute probabilities in the ratio $p / p s^{t}$ are lower than in $1 / s^{t}$, implying that the ratio of probability weights attached to the former pair is closer to unity, i.e.

$$
\frac{w(1)}{w\left(s^{t}\right)}>\frac{w(p)}{w\left(p s^{t}\right)}
$$

holds for subproportional weights $w$. Hence,

$$
\frac{v(x) w(1)}{v(y) \rho^{t} w\left(s^{t}\right)}>\frac{v(x) w(p)}{v(y) \rho^{t} w\left(p s^{t}\right)},
$$

implying that the difference in the subjective valuations of the immediate and the delayed rewards shrinks when outcomes are made risky, which reduces the relative attractiveness of the immediate reward $x$.

A similar argument can be applied to another puzzling phenomenon discovered by Noussair and $\mathrm{Wu}$ (2006), delay dependence of risk tolerance. They report that subjects, when choosing between relatively safe and risky gambles realized in the present as well as three months into the future, are more risk tolerant for the future gambles. This effect does not disappear when the gambles are played out in the near future rather than in the present. According to our approach to time discounting, a change in relative risk tolerance with respect to simple risky prospects is a direct consequence of subproportionality. To see this, assume that the decision maker is faced with a choice between $(x, p)$ versus $(y, q)$ with $p>q$ and $y>x$. Subproportionality implies for $t>1$,

$$
\frac{w(p)}{w(q)}>\frac{w(p s)}{w(q s)}>\frac{w\left(p s^{t}\right)}{w\left(q s^{t}\right)}
$$

\footnotetext{
${ }^{3}$ For an alternative explanation see Fudenberg and Levine (2009).
} 
and, therefore,

$$
\frac{v(x) w(p)}{v(y) w(q)}>\frac{v(x) \rho w(p s)}{v(y) \rho w(q s)}>\frac{v(x) \rho^{t} w\left(p s^{t}\right)}{v(y) \rho^{t} w\left(q s^{t}\right)} .
$$

By moving risky outcomes into the more remote future, the absolute levels of their subjective probabilities decrease commensurately, without their ratio being changed. Due to subproportionality, the corresponding probability weights become less and less distinguishable from each other as time passes, thereby reducing the difference in relative valuations. Hence, a preference for the comparatively less risky gamble $(x, p)$ gets weaker over time.

Finally, accounting for uncertainty in the proposed way gives rise to subadditivity, an additional feature of time discounting documented in the empirical literature (Read, 2001). Whereas decreasing impatience refers to a delay with respect to the present, subadditivity pertains to period length, irrespective of where the period is located on the timeline. Discounting behavior is subadditive when the product of the discount factors resulting from a partition of a specific interval is smaller than the discount factor applied to the interval at once. This characteristic of time discounting likewise follows from subproportionality of probability weights: Suppose that $\left(\Delta_{i}\right)_{i=1, \ldots, n}$ is a partition of delay $D$ such that $D=\sum_{i=1}^{n} \Delta_{i}$. Since subproportionality implies $w(p) w(q)<w(p q)$ (see Equation 1), $(w(p) w(q)) w(z)<w(p q) w(z)<w(p q z)$ holds for any probabilities $0<p, q, z<1$, and

$$
\prod_{i=1}^{n} w\left(s^{\Delta_{i}}\right)<w\left(\prod_{i=1}^{n} s^{\Delta_{i}}\right)=w\left(s^{D}\right)
$$

follows by induction. Consequently, when partitioning a specific time interval, the product of the corresponding weights of the survival probabilities $\prod_{i=1}^{n} w\left(s^{\Delta_{i}}\right)$ is smaller than the weight attached to the probability of survival over the whole period $w\left(\prod_{i=1}^{n} s^{\Delta_{i}}\right)$ and, hence, the product of the discount factors associated with the partition is smaller as well.

\subsection{The Effects of Uncertainty on Hyperbolicity}

Another important insight from our approach concerns the direct impact of uncertainty on discounting behavior: Increasing uncertainty not only raises the level of discount rates but also exacerbates short-term impatience. A formal proof is included in Appendix A.4. Panels A2 to C2 in Figure I illustrate this effect for $\alpha=0.5$, a medium-sized departure from linear probability weighting, and $r=0.1$. When the survival probability $s$ declines from 0.8 to 0.5 , the resulting discount function departs more strongly from exponentiality as Panel B2 shows. Hence, the decrease in impatience associated with higher uncertainty is more pronounced as well (Panel C2). In the numerical example here, the discount rate of an exponential discounter would rise from 0.32 to 0.79 , representing the well-known 
level effect of rising uncertainty (see Appendix A.1).

This analysis implies that hyperbolicity becomes more pronounced when intrinsic uncertainty increases. This uncertainty may stem from different sources. At the personal level, the survival probability $s$ can be interpreted literally and, consequently, older or ailing people are more likely to exhibit stronger short-run impatience than younger and healthy ones ceteris paribus. Another important channel through which uncertainty may influence individuals' decisions is the institutional environment. In environments where property rights are only weakly protected or institutions of contract enforcement are not reliable, the probability of contract survival will be perceived to be rather low. Therefore, our framework predicts that in countries with weak institutions, such as many developing countries, excessive short-run impatience should be prevalent, which seems to be supported by empirical evidence (Poulos and Whittington, 2000).

If intrinsic uncertainty in conjunction with nonlinear probability weighting is such a powerful concept for explaining anomalies in time discounting, the crucial question remains whether risk taking behavior indeed predicts time discounting behavior at the level of the individual decision maker. For this purpose, we conducted an experiment with salient monetary incentives, described in detail in the next section.

\section{Experimental Design}

The experiment took place at the Institute for Empirical Research in Economics (IERE), University of Zurich, in May 2006. Participants were recruited from the IERE subject pool, which consists of students from all fields offered at the University of Zurich and the Swiss Federal Institute of Technology Zurich. In total, we analyzed 114 subject's responses. ${ }^{4}$ The experiment consisted of two main parts, one dedicated to eliciting certainty equivalents for risky prospects, the other one to eliciting future equivalents and their corresponding imputed discount rates for temporal prospects. ${ }^{5}$

We used similar procedures to elicit certainty equivalents and discount rates, in order to economize on subjects' cognitive effort. For both types of tasks we implemented choice menus containing a list of 20 varying alternatives which had to be judged against a fixed option. To familiarize subjects with the nature of the procedure, the instructions contained examples and trial problems. The experimenters checked the choices in the

\footnotetext{
${ }^{4}$ We omitted four subjects' responses from our analysis, since these subjects reported that they would not be able to cash in their delayed payments at the respective payment dates. Three of them would have been on vacation then, the fourth person had planned a long visit abroad. Hence, their choices were not informative of their time preferences.

${ }^{5}$ Instructions are available upon request. The experiment was programmed and conducted with the software z-Tree (Fischbacher, 2007).
} 
trial problems to verify that the subjects had comprehended the task. Besides a show up fee of $\mathrm{CHF} 10$ (CHF $1 \approx$ USD 0.8 at the time of the experiment), each subject was paid according to one of her risky choices and one of her temporal choices selected randomly at the very end of the experiment. Subjects received their compensation for the risky choices in cash immediately after completion of all the tasks. The compensation for their intertemporal choices was paid out to them at the respective dates when they cashed in vouchers issued to them at the end of the experiment. Payment modalities are described in detail below. Subjects could work at their own speed. On average, it took them 1.25 hours to complete the experiment, including a socioeconomic questionnaire presented after the choice tasks.

\subsection{Elicitation of Certainty Equivalents}

Since the objective of the risk task was generating observations on certainty equivalents, on the basis of which individual probability weights could be estimated, a fairly large number of observations per person was needed. To obtain individual lottery evaluations, subjects were presented with 20 choice menus, each one involving a specific binary lottery $\mathcal{L}=\left(x_{1}, p ; x_{2}\right)$, with $x_{1}>x_{2} \geq 0$, labelled Option $A$ in Figure II. Option $B$ in the choice menu represented the guaranteed alternatives, ranging from the higher lottery outcome $x_{1}$ to the lower outcome $x_{2}$. Every subject had to choose her preferred option in each row of the choice menu. In Figure II, a hypothetical subject prefers all guaranteed payments larger than CHF 36 to the stated lottery, and prefers the risky option in the remaining rows. This subject's valuation of the lottery, her certainty equivalent ce, is calculated as the arithmetic mean of the two amounts next to her indifference point, amounting to CHF 37 in the example here.

The set of lotteries, listed in Table I, included a wide range of outcomes and probabilities. Every subject was confronted with this set of lotteries once. To avoid order effects, the choice menus appeared in an individualized random order.

At the end of the experiment, after the subject had completed all the tasks, one row of one choice menu was randomly selected for each payment. If the subject had opted for the lottery there, her decision was played out for real. If the subject had opted for the guaranteed payoff, the respective amount was paid out to her. On average, subjects earned CHF 36.60 in cash for the risk task, including the show-up fee of CHF 10, to be paid out immediately. Cash payments for the risk task were considerably higher than the local student assistant's hourly wage. 
Figure II: Choice Menu — Risk

\begin{tabular}{|c|c|c|c|c|c|c|}
\hline & Option A & \multicolumn{4}{|c|}{ Your Choice } & \multirow{2}{*}{$\begin{array}{c}\begin{array}{c}\text { Option B } \\
\text { (guaranteed reward) }\end{array} \\
\text { CHF } 50\end{array}$} \\
\hline 1 & \multirow{20}{*}{$\begin{array}{c}\text { Gain } \\
\text { of CHF } 50 \text { with a } \\
\text { probability of } \\
75 \%\end{array}$} & $\mathrm{~A}$ & 0 & 0 & B & \\
\hline 2 & & A & 0 & 0 & B & CHF 48 \\
\hline 3 & & A & 0 & 0 & B & CHF 46 \\
\hline 4 & & A & 0 & 0 & B & CHF 44 \\
\hline 5 & & A & 0 & 0 & B & CHF 42 \\
\hline 6 & & A & 0 & 0 & B & CHF 40 \\
\hline 7 & & A & 0 & 0 & B & CHF 38 \\
\hline 8 & & A & 0 & 0 & B & CHF 36 \\
\hline 9 & & A & 0 & 0 & B & CHF 34 \\
\hline 10 & & A & 0 & 0 & B & CHF 32 \\
\hline 11 & & A & 0 & 0 & B & CHF 30 \\
\hline 12 & & A & 0 & 0 & B & CHF 28 \\
\hline 13 & & A & 0 & 0 & B & CHF 26 \\
\hline 14 & & A & 0 & 0 & B & CHF 24 \\
\hline 15 & & A & 0 & 0 & B & CHF 22 \\
\hline 16 & & A & 0 & 0 & B & CHF 20 \\
\hline 17 & & A & 0 & 0 & B & CHF 18 \\
\hline 18 & & A & 0 & 0 & B & CHF 16 \\
\hline 19 & & A & 0 & 0 & B & CHF 14 \\
\hline 20 & & A & 0 & 0 & B & CHF 12 \\
\hline
\end{tabular}

Table I: Risky Prospects

\begin{tabular}{cccccc}
\hline \hline$p$ & $x_{1}$ & $x_{2}$ & $p$ & $x_{1}$ & $x_{2}$ \\
\hline 0.1 & 20 & 10 & 0.25 & 50 & 20 \\
0.5 & 20 & 10 & 0.5 & 50 & 20 \\
0.9 & 20 & 10 & 0.75 & 50 & 20 \\
0.05 & 40 & 10 & 0.95 & 50 & 20 \\
0.25 & 40 & 10 & 0.05 & 150 & 50 \\
0.5 & 40 & 10 & 0.5 & 10 & 0 \\
0.75 & 40 & 10 & 0.5 & 20 & 0 \\
0.95 & 40 & 10 & 0.05 & 40 & 0 \\
0.05 & 50 & 20 & 0.95 & 50 & 0 \\
0.1 & 150 & 0 & 0.25 & 40 & 0 \\
\hline \hline
\end{tabular}

Outcomes $x_{1}$ and $x_{2}$ are stated in CHF, $p$ denotes the probability of $x_{1}$ materializing. 


\subsection{Elicitation of Discount Rates}

The second task served to elicit individual discount rates. We elicited preferences in a manner equivalent to the risk procedure for three temporal prospects, summarized in Table II. The choice menus, designed as in Figure III, contained 20 binary choices each ${ }^{6}$. Subjects had to choose between a certain earlier payment (at $\left.t_{0}\right)$ and a certain later payment (at $t_{1}$ ). Specifically, Option A represented a fixed earlier amount of CHF 60, and Option $B$ offered 20 alternative amounts delayed by two months and four months, respectively. The varying alternatives were sorted in descending order from the highest amount to the lowest amount. The delayed amounts incorporated an interest payment at a simple annualized rate of $\delta \in[0 \%, 95 \%]$ over the corresponding time interval $\left[t_{0}, t_{1}\right]$. These rates were exhibited in the right-most column of the choice menu. The principal amount of CHF 60 and the range of interest rates were chosen to provide salient incentives, so that deferring the reward was actually worthwhile. The arithmetic mean of the two monetary amounts next to the indifference point on the choice menu provided the imputed discount rate $\delta_{t_{0} t_{1}}$. For the hypothetical subject in Figure III, for instance, the future equivalent amounts to CHF 70.50, implying a discount rate of $52.5 \%$ per annum.

Table II: Temporal Prospects

\begin{tabular}{cllc}
\hline \hline Amount & Earlier payment at $t_{0}$ & Later payment at $t_{1}$ & Discount rate $\delta_{t_{0} t_{1}}$ \\
\hline$y_{t_{0}}=60$ & next day & in 2 months +1 day & $\delta_{02}$ \\
$y_{t_{0}}=60$ & next day & in 4 months +1 day & $\delta_{04}$ \\
$y_{t_{0}}=60$ & in 2 months +1 day & in 4 months +1 day & $\delta_{24}$ \\
\hline \hline
\end{tabular}

We applied a similar random payment method in the time task as in the risk task: One of each subject's choices was paid out for real at the corresponding payment date. Average payoffs for the time task amounted to CHF 64.20. Therefore, total average payments for both risk and time tasks summed to more than four times students' opportunity costs, measured by the student assistants' hourly wages.

Since our objective was to elicit discount rates over certain payments, we took special care with the payment procedure: First, every single subject got paid for one of her intertemporal choices all of which entailed a payment of the same order of magnitude. Not paying off everyone may render the stochastic nature of the experimental earnings salient and interfere with the objective of eliciting discount rates over guaranteed amounts of money. ${ }^{7}$ The second issue concerns the credibility of payment. In order to control for uncertainty arising from subjects' doubts about experimenter reliability, an official

\footnotetext{
${ }^{6}$ Coller and Williams (1999) propose a similar setup.

${ }^{7}$ It is reasonable to assume that subjects did not integrate the probability of a specific prospect
} 
Figure III: Choice Menu — Time

\begin{tabular}{|c|c|c|c|c|c|c|c|}
\hline \multirow[b]{2}{*}{1} & \multirow{2}{*}{$\begin{array}{c}\text { Option A } \\
\text { payment tomorrow }\end{array}$} & \multicolumn{4}{|c|}{ Your Choice } & \multicolumn{2}{|c|}{$\begin{array}{c}\text { Option B } \\
\text { payment in } 4 \text { months }+1 \text { day }\end{array}$} \\
\hline & & A & 0 & 0 & B & CHF 79 & $95 \%$ \\
\hline 2 & \multirow{19}{*}{$\begin{array}{c}\text { Amount } \\
\text { of CHF } 60\end{array}$} & A & 0 & 0 & B & CHF 78 & $90 \%$ \\
\hline 3 & & A & 0 & 0 & B & CHF 77 & $85 \%$ \\
\hline 4 & & A & 0 & 0 & B & CHF 76 & $80 \%$ \\
\hline 5 & & A & 0 & 0 & B & CHF 75 & $75 \%$ \\
\hline 6 & & $\mathrm{~A}$ & 0 & 0 & $\mathrm{~B}$ & CHF 74 & $70 \%$ \\
\hline 7 & & A & 0 & 0 & B & CHF 73 & $65 \%$ \\
\hline 8 & & A & 0 & 0 & B & CHF 72 & $60 \%$ \\
\hline 9 & & $\mathrm{~A}$ & 0 & 0 & $\mathrm{~B}$ & CHF 71 & $55 \%$ \\
\hline 10 & & A & 0 & 0 & B & CHF 70 & $50 \%$ \\
\hline 11 & & A & 0 & 0 & B & CHF 69 & $45 \%$ \\
\hline 12 & & $\mathrm{~A}$ & 0 & 0 & $\mathrm{~B}$ & CHF 68 & $40 \%$ \\
\hline 13 & & $\mathrm{~A}$ & 0 & 0 & B & CHF 67 & $35 \%$ \\
\hline 14 & & $\mathrm{~A}$ & 0 & 0 & B & CHF 66 & $30 \%$ \\
\hline 15 & & A & 0 & 0 & B & CHF 65 & $25 \%$ \\
\hline 16 & & A & 0 & 0 & B & CHF 64 & $20 \%$ \\
\hline 17 & & A & 0 & 0 & B & CHF 63 & $15 \%$ \\
\hline 18 & & A & 0 & 0 & B & CHF 62 & $10 \%$ \\
\hline 19 & & A & 0 & 0 & B & CHF 61 & $5 \%$ \\
\hline 20 & & $\mathrm{~A}$ & 0 & 0 & B & CHF 60 & $0 \%$ \\
\hline
\end{tabular}


voucher of the Swiss Federal Institute of Technology Zurich, an institution with highest credit ratings, was issued to them. This payment method was explained in detail in the instructions, and a specimen of the voucher was included in the instruction set. This essentially rules out that subjects' subjective uncertainty originated from potential contract breach.

A third possibly confounding factor are transaction costs. Transaction costs should be the same regardless of the payment date in order to avoid inducing present bias resulting from immediately available cash payments. Therefore, even those subjects entitled to the immediate amount of CHF 60 received a voucher and had to make a trip to the Swiss Federal Institute of Technology the next day. ${ }^{8}$

\section{Econometric Specification}

The data elicited in the experiment provide two types of main variables: certainty equivalents ce for risky prospects, and imputed discount rates $\delta_{t_{0} t_{1}}$ for temporal prospects. We first discuss our econometric approach to risky choice and, subsequently, describe the method employed to test our central hypothesis.

\subsection{Behavior under Risk}

Modeling decisions under risk encompasses two components, a model of behavior on the one hand, and assumptions regarding decision errors on the other hand. Risk taking behavior is modeled by a flexible functional in the spirit of prospect theory $(\mathrm{PT})$.

According to $\mathrm{PT}$, an individual values a two-outcome lottery $\mathcal{L}=\left(x_{1}, p ; x_{2}\right)$, where $x_{1}>x_{2} \geq 0$, by

$$
w(p) v\left(x_{1}\right)+(1-w(p)) v\left(x_{2}\right) .
$$

The function $v(x)$, with $v(0)=0$ and $v^{\prime}(x)>0$, describes how monetary outcomes $x$ are valued. The function $w(p)$ assigns a subjective weight to every outcome probability $p$, with $w(0)=0, w(1)=1$, and $w^{\prime}(p)>0$. The decision maker's predicted certainty equivalent $\hat{c e}$ for this lottery can then be written as

$$
\hat{c e}=v^{-1}\left[w(p) v\left(x_{1}\right)+(1-w(p)) v\left(x_{2}\right)\right] .
$$

being chosen for payment, which equals $1 / 3$ here, directly with their subjective probability of contract survival (see Hey and Jinkwon (2005) for supporting evidence in the case of risk taking). If they did so, however, by additionally discounting future utility by $w(1 / 3)$, such that $D(t)=\rho^{t} w(1 / 3) w\left(s^{t}\right)$, only the magnitude of discount rates would have been affected and all of our results on decreasing impatience still go through.

${ }^{8}$ All the sessions took place in the afternoon after the end of opening hours of the cashier's desk. 
In order to make PT operational, we have to assume specific functional forms for the value function $v(x)$ and the probability weighting function $w(p)$. A natural candidate for $v(x)$ is the power function

$$
v(x)=x^{\eta}, x \geq 0, \eta>0,
$$

which has turned out to be the best compromise between parsimony and goodness of fit in the context of prospect theory (Stott, 2006).

A variety of functions for modeling probability weights $w(p)$ have been proposed in the literature (Karmarkar, 1979; Lattimore, Baker, and Witte, 1992; Tversky and Kahneman, 1992; Prelec, 1998). Since our primary interest lies in subproportionality, we focus on the one-parameter specification proposed by Prelec (1998), as defined in Equation 4.

The functions' single parameter $\alpha$ is equivalent to its slope at the inflection point. Smaller values of $\alpha$ reflect a more subproportional and more S-shaped curve. Our main hypothesis can now be expressed in terms of the weighting function parameter $\alpha$ : We predict that $\alpha$ is negatively correlated with the size of the observed change in discount rates, i.e. the greater the departure from linear probability weighting, the larger the decline in discount rates.

With regard to error specification we have to reconsider our measurement procedure. In the course of the experiment, an individual's risk taking behavior was captured by her certainty equivalents $c e_{l}$ for a set of 20 different lotteries $\mathcal{L}_{l}=\left(x_{1 l}, p_{l} ; x_{2 l}\right), l \in\{1, \ldots, 20\}$. Since PT explains deterministic choice, actual certainty equivalents $c e_{l}$ are bound to deviate from the predicted certainty equivalents $\hat{c e}_{l}$ by an error $\epsilon_{l}$, which has to be taken account of. Therefore, we assume that the observed certainty equivalents $c e_{l}$ can be expressed as $c e_{l}=\hat{c}_{l}+\epsilon_{l}$. The Central Limit Theorem supports our assumption that errors are normally distributed and simply add white noise.

Furthermore, we account for heteroskedasticity in the error variance: For each lottery subjects had to consider 20 certain outcomes, equally spaced throughout the lottery's outcome range $x_{1 l}-x_{2 l}$. Since the observed certainty equivalent $c e_{l}$ is calculated as the arithmetic mean of the smallest certain amount preferred to the lottery and the subsequent certain amount, the error is proportional to the outcome range. Therefore, the standard deviation $\nu_{l}$ of the error term distribution has to be normalized by the outcome range, yielding $\nu_{l}=\nu\left(x_{1 l}-x_{2 l}\right)$, where $\nu$ denotes an additional parameter to be estimated. In total, therefore, three parameters per subject were estimated by maximum likelihood: the curvature of the value function $\eta$, the curvature of the probability weighting function $\alpha$, and the normalized standard deviation of the decision error $\nu$. 


\subsection{Behavior over Time}

Subjects' responses to the intertemporal choice tasks in the experiment provided us with measurements of discount rates $\delta_{02}$ and $\delta_{04}$, imputed from the intertemporal tradeoffs between present payments and payments delayed by two and four months, respectively. However, our main hypothesis links the degree of departure from linear probability weighting to the change in the utility equalizing discount rates. Clearly, these deviate from the observed discount rates unless $v$ is linear. While the curvature of the utility function $v$ cannot induce decreasing impatience by itself, it certainly does have a confounding effect on the magnitude of the change in the measured discount rates $\Delta \delta=\delta_{02}-\delta_{04}$ : In the presence of nonlinear probability weighting, $\Delta \delta$ gets amplified by the concavity of the utility function (see Appendix A.2). Specifically, the more concave $v$, the larger the measured difference in the discount rates $\Delta \delta$ if $w(p)$ is not linear. Therefore, we have to control for the degree of concavity $\eta$ in the regression model.

\subsection{Regression Model}

We investigate the hypothesized relationship between decreasing impatience and probability weighting by regressing the difference between the imputed discount rates $\delta_{02}$ and $\delta_{04}, \Delta \delta$, on a vector of regressors $c$. In the base model, Model 1 , the vector $c$ consists of a constant and the individuals' estimated risk preference parameters: $\eta$ captures concavity of the value function, $\alpha$ the curvature of the probability weighting function. Additionally, we estimate an extended version of the base model, Model 2, by controlling for a set of individual characteristics. In particular, these controls comprise gender (labeled Female), age (Age), the logarithm of disposable income per month (Log-Income), a binary variable indicating whether the subject is familiar with investment decisions (Experience) as well as the test score for the Cognitive Reflection Test (CRT) (Frederick, 2005). This three-question test measures specific aspects of cognitive ability. ${ }^{9}$

Unlike the exemplary choice pattern displayed in Figure III, a decision maker may have opted for the same option in all rows of the choice menu, which results in a censored observation. In particular, she may have always preferred the smaller sooner option, indicating that her discount rate may lie beyond the maximum value of $95 \% .{ }^{10}$ As a consequence, the difference between the observed discount rates $\delta_{02}$ and $\delta_{04}$ is affected by censoring as well. As ordinary least square (OLS) yields biased estimates in this case, we account for this issue by a censored regression model, described in detail in Appendix B.

\footnotetext{
${ }^{9}$ Summary statistics of the controls are included in Appendix E, Table A.1.

${ }^{10} \mathrm{~A}$ decision maker may also always prefer the later larger option. In this case, we assume a discount rate of $0 \%$. The number of observations at the boundary of the choice menu are listed in Table A.2 of Appendix E.
} 
The model has the following form:

$$
\Delta \delta_{i}^{*}=c_{i} \underbrace{\left(\beta_{02}-\beta_{04}\right)}_{\Delta \beta}+\underbrace{e_{02, i}-e_{04, i}}_{\Delta e_{i}}
$$

where $\Delta \delta_{i}^{*}$ specifies the true, but potentially unobserved, difference between $\delta_{02}$ and $\delta_{04}$ for individual $i, i \in\{1, \ldots, 114\}$. The error term $\Delta e_{i}$ is normally distributed with mean zero and variance $\sigma^{2}$. The interpretation of the regression coefficients $\Delta \beta$ is equivalent to those of OLS regression, also displayed in the regression output (Table IV below).

\section{Results}

In the following section we analyze the raw data on risk taking behavior and time discounting, and present the estimates for subjects' probability weights. Finally, we examine our central hypothesis on the relationship between subjects' sensitivities with respect to changes in probability and delay.

\subsection{Descriptive Analysis}

For the domain of risk taking, Figure IV summarizes observed behavior by the median relative risk premia $R R P=(e v-c e) /|e v|$, where $e v$ denotes the expected value of a lottery's payoff and ce stands for the observed certainty equivalent. $R R P>0$ indicates risk aversion, $R R P<0$ risk seeking, and $R R P=0$ risk neutrality. The median relative risk premia, sorted by the probability $p$ of the higher gain, show a systematic relationship between aggregated risk attitudes and lottery probabilities: Subjects' choices display the familiar pattern, i.e. they are risk averse for high-probability gains, but risk seeking for low-probability gains, supporting our assumptions.

As far as intertemporal choices are concerned, people's average behavior exhibits decreasing impatience, i.e. subjects discount less remote outcomes more strongly than more remote ones: The first column in Table III reveals that the discount rates imputed from subject's choices decline on average by 7 percentage points per annum when the time horizon is extended from two months to four months. The average data veil heterogeneity as well as the extent of decreasing impatience, however. Whereas the majority of approximately $54 \%$ of all subjects exhibit decreasing discount rates over time, $\Delta \delta>0$ (second column), about $29 \%$ exhibit constant discount rates (third column), and the residual group reveals increasing discount rates (fourth column). ${ }^{11}$ Average discount

\footnotetext{
${ }^{11}$ This composition of discounting types resembles the one found in a representative field experiment conducted with 500 adults from the German population (Dohmen, Falk, Huffman, and Sunde, 2007).
} 


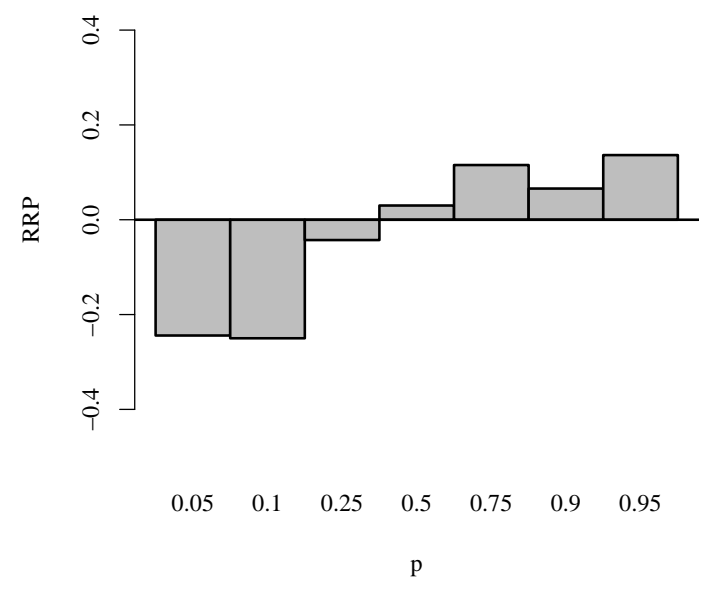

rates for those subjects that exhibit decreasing impatience amount to $\delta_{02}=46 \%$ p.a. and $\delta_{04}=30 \%$ p.a., respectively, reflecting a greater change than do the overall averages. For the group with constant impatience, $\Delta \delta=0$, the average discount rate amounts to $21 \%$, which is significantly lower than the overall average (Wilcoxon rank-sum test: $p$-value $<0.002){ }^{12}$

Table III: Average Discount Rates and Risk Parameters

\begin{tabular}{ccccc}
\hline \hline & & \multicolumn{3}{c}{ Subjects with } \\
\cline { 3 - 5 } & all & $\Delta \delta>0$ & $\Delta \delta=0$ & $\Delta \delta<0$ \\
& $100 \%$ & $54.4 \%$ & $28.9 \%$ & $16.7 \%$ \\
\hline$\delta_{02}$ & 0.366 & 0.459 & & 0.328 \\
& $(0.025)$ & $(0.022)$ & 0.213 & $(0.024)$ \\
$\delta_{04}$ & 0.296 & 0.302 & $(0.024)$ & 0.418 \\
& $(0.023)$ & $(0.019)$ & & $(0.028)$ \\
\hline$\Delta \delta$ & 0.070 & 0.157 & 0 & -0.090 \\
& $(0.014)$ & $(0.011)$ & & $(0.008)$ \\
\hline$\alpha$ & 0.499 & 0.424 & 0.565 & 0.633 \\
& $(0.023)$ & $(0.020)$ & $(0.022)$ & $(0.026)$ \\
$\eta$ & 1.022 & 1.018 & 0.989 & 1.090 \\
& $(0.033)$ & $(0.034)$ & $(0.036)$ & $(0.025)$ \\
\hline \hline
\end{tabular}

Standard errors in parentheses. Excluding 24 subjects with censored observations $(n=90)$.

Furthermore, average observed behavior also exhibits subadditivity of discount rates,

\footnotetext{
${ }^{12}$ The distributions of the observed discount rates are shown in Appendix C.
} 
consistent with our theoretical framework. For the 87 subjects with three non-censored observations, the discount factor over the whole period of four months equals 0.917 and, thus is considerably higher than the product of the discount factors over the respective two-month subperiods amounting to 0.905 (Wilcoxon signed rank test, $p$-value $<0.0003$ ).

\subsection{Risk Preference Parameters}

Whereas one of our central variables, changing impatience $\Delta \delta$, is directly observable, the other one, departure from linear probability weighting, has to be estimated from our data on certainty equivalents.

Individual risk preference parameters $\eta$ and $\alpha$ were estimated on the basis of the econometric model discussed in Section 4.1. As Table III reveals, the value of the curvature parameter $\eta$ of the utility function, 1.022, is not distinguishable from unity on average, with little variation across discounting types. The average subproportionality index $\alpha$ amounts to 0.499 , indicating a pronounced departure from linear probability weighting in line with previous findings (Tversky and Kahneman, 1992; Gonzalez and Wu, 1999; Bruhin, Fehr-Duda, and Epper, 2007). ${ }^{13}$

The overall picture revealed by our data is consistent with the typical empirical findings: On average, subjects systematically violate linear probability weighting and constant discounting. But the central question, namely whether the extent of the departure from linear probability weighting empirically predicts the degree of hyperbolicity of discounting behavior has yet to be answered.

\subsection{Probability Weighting and Decreasing Impatience}

With the estimates of people's probability weighting parameters and their observed discount rates we are now equipped to examine our central hypothesis, namely whether nonlinear probability weighting predicts decreasing impatience at the level of individual behavior. A first indication that our conjecture bears out can be found in Table III. The average estimated subproportionality index $\alpha$ varies substantially across discounting types and exhibits a systematic pattern: $\alpha$ is lowest for the group with decreasing impatience and highest for the group with increasing impatience.

This finding is confirmed by the estimates of the regression models. Table IV displays the results derived by OLS as well as by the censored regression method. Inspection of the coefficients indicates that censoring seems not to be an important problem: When omitting the 24 censored observations, OLS yields coefficients very close to the estimates of the censored regression model. Furthermore, both specifications (Models 1 and 2)

\footnotetext{
${ }^{13}$ Histograms of the parameter distributions are included in Appendix D.
} 
lead to the same conclusion: Consistent with our central hypothesis, the curvature of the probability weighting function is a significant determinant of decreasing impatience. Table IV shows the estimated coefficient of $\alpha$ to be approximately -0.2 , which remains robust to inclusion of additional controls. All the respective estimates are significant at the 1\%-level. The effect is not only highly significant, it is also quite substantial: A decrease in the subproportionality index $\alpha$ by 0.1 is associated with an increase in $\Delta \delta$ by 2 percentage points per annum.

Table IV: Regression Results

Dependent Variable: $\Delta \delta\left(\Delta \delta^{*}\right)$

\begin{tabular}{|c|c|c|c|c|}
\hline & \multicolumn{2}{|c|}{$\mathrm{OLS}^{a}$} & \multicolumn{2}{|c|}{ Censored } \\
\hline & Model 1 & Model 2 & Model 1 & Model 2 \\
\hline Intercept & $\begin{array}{r}0.105 \\
(0.056)\end{array}$ & $\begin{array}{c}0.153 \\
(0.221)\end{array}$ & $\begin{array}{r}0.091 \\
(0.053)\end{array}$ & $\begin{array}{r}0.132 \\
(0.208)\end{array}$ \\
\hline$\eta$ & $\begin{array}{r}0.065 \\
(0.042)\end{array}$ & $\begin{array}{r}0.053 \\
(0.045)\end{array}$ & $\begin{array}{r}0.073 \\
(0.040)\end{array}$ & $\begin{array}{r}0.060 \\
(0.044)\end{array}$ \\
\hline$\alpha$ & $\begin{array}{r}-0.202^{* *} \\
(0.067)\end{array}$ & $\begin{array}{r}-0.221^{* *} \\
(0.074)\end{array}$ & $\begin{array}{r}-0.176^{* *} \\
(0.062)\end{array}$ & $\begin{array}{r}-0.199^{* *} \\
(0.074)\end{array}$ \\
\hline Female & & $\begin{array}{l}-0.013 \\
(0.030)\end{array}$ & & $\begin{array}{l}-0.012 \\
(0.031)\end{array}$ \\
\hline Age & & $\begin{array}{l}-0.001 \\
(0.006)\end{array}$ & & $\begin{array}{l}-0.001 \\
(0.007)\end{array}$ \\
\hline Log-Income & & $\begin{array}{l}-0.010 \\
(0.023)\end{array}$ & & $\begin{array}{l}-0.007 \\
(0.022)\end{array}$ \\
\hline Experience & & $\begin{array}{r}0.016 \\
(0.031)\end{array}$ & & $\begin{array}{r}0.018 \\
(0.030)\end{array}$ \\
\hline$C R T$ & & $\begin{array}{r}0.023 \\
(0.017)\end{array}$ & & $\begin{array}{r}0.022 \\
(0.016)\end{array}$ \\
\hline$\hat{\sigma}$ & 0.121 & 0.122 & 0.083 & 0.083 \\
\hline$R^{2}$ or (LogLik) & 0.142 & 0.177 & $(46.635)$ & $(49.276)$ \\
\hline Observations & 90 & 90 & 114 & 114 \\
\hline Parameters & 3 & 8 & 7 & 17 \\
\hline $\begin{array}{l}\text { a) without censor } \\
\text { strapped standard } \\
\text { strapping account } \\
\text { estimated quantit }\end{array}$ & $\begin{array}{l}\text { observatic } \\
\text { rrors in pa } \\
\text { or the fact } \\
\text {. }\end{array}$ & $\begin{array}{l}{ }^{* *} \text { signifi } \\
\text { theses }(10 \\
\text { at the reg }\end{array}$ & $\begin{array}{l}\text { at the } 19 \\
\text { replicati } \\
\text { ss. } \alpha \text { and }\end{array}$ & $\begin{array}{l}\text { vel. Boot- } \\
\text {. Boot- } \\
\text { are }\end{array}$ \\
\hline
\end{tabular}

The coefficients of the curvature parameter $\eta$, however, are not statistically different from zero. This result is consistent with our hypothesis that utility curvature per se does not impact the extent of decreasing impatience. Furthermore, none of the other individual characteristics show a significant effect.

A likelihood ratio test of the censored Model 2 against Model 1 renders a $p$-value of 0.954 , favoring the more parsimonious Model 1 , as the controls do not substantially 
contribute to explaining the variance in $\Delta \delta^{*}$. Furthermore, the regression models explain a rather large fraction of total variance: Model 1 estimated by OLS, for instance, yields an $R$-squared value of $14.2 \%{ }^{14}$ These findings present conclusive evidence that comparatively more subproportional probability weighting is associated with a stronger decline in discount rates.

\subsection{Perceived Uncertainty and the Pure Rate of Time Prefer- ence}

In sum, our analysis provides support for the hypothesized relationship between probability weighting and decreasing impatience. Our framework predicts such a relationship ceteris paribus, holding constant the other model parameters, specifically the subjective probability of contract survival $s$ and the pure rate of time preference $r$, both of which are not observable. In our experimental setting with decisions over a short time horizon, the subjective probability of contract survival $s^{t}$ should lie very close to unity since mortality risk is very low in our age group of subjects and we took great care to communicate experimenter reliability. One way of checking the plausibility of our model is to investigate whether actual choices are indeed consistent with this conjecture, i.e. whether values of $s$ implied by our data lie in the vicinity of one for a wide range of plausible values of the pure rate of time preference $r$.

For this purpose, we examine the combinations of $s$ and $r$ that are consistent with the observed intertemporal tradeoffs between more immediate and more remote payments $y_{t_{0}}$ and $y_{t_{1}}$. We solve for all feasible combinations of $\tilde{s}$ and $\tilde{t}$ that are compatible with the observed choices by inserting the estimates for subjects' average behavioral parameters $\eta$ and $\alpha$ into Equation 2. As is clear from Equation 2, a higher probability of contract survival needs to be compensated by a higher pure rate of time preference, ceteris paribus, to keep individuals indifferent between more immediate and more remote rewards.

As Figure $\mathrm{V}$ shows, the feasible $(\tilde{s}, \tilde{r})$-combinations indeed exhibit a rising profile, with $\tilde{s}$ starting out at slightly below $96 \%$ p.a. and converging to $100 \%$, when the pure rate of time preference increases from $0 \%$ to $20 \%$ p.a. and beyond. What this exercise shows is that the data, interpreted within our framework, are consistent with a very high subjective probability that contracts survive at least one year, in accordance with our assumptions. Furthermore, accounting for inherent uncertainty implies rates of pure time preference in a plausible range lying considerably below the respective range of observed discount rates.

\footnotetext{
${ }^{14}$ Moreover, it can be shown that estimates are robust to an alternative specification of the probability weighting curve (see Appendix F) as well as to restricting observations to subsamples.
} 
Figure V: Feasible $(\tilde{s}, \tilde{r})$-Combinations

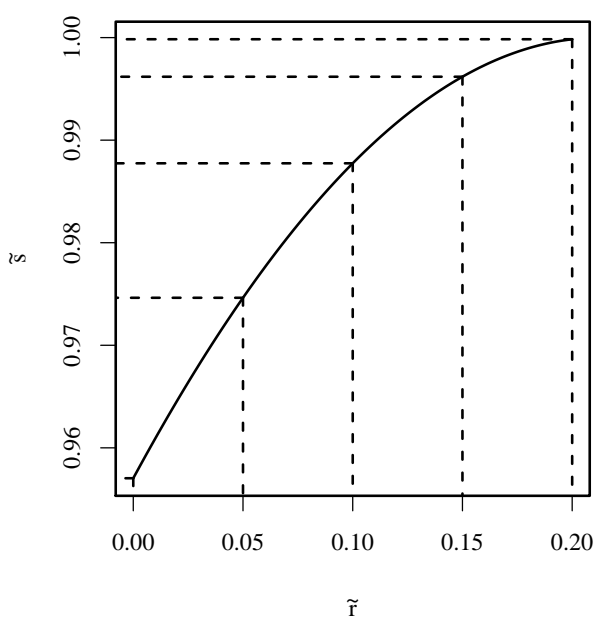

This suggests that even allegedly guaranteed future outcomes are viewed as slightly uncertain, in line with direct questionnaire evidence provided in Patak and Reynolds (2007). The authors asked respondents to rate their certainties for the same rewards, delayed by $1,2,30,180$, and 365 days, respectively, which they had encountered during the preceding choice experiment. The respondents reported ratings that clearly declined with the length of delay. Moreover, using a similar method, Takahashi, Ikeda, Hasegawa, and Greene (2007) found that such subjective probabilities of obtaining delayed rewards decay in a hyperbolic-like manner, consistent with the hyperbolic decline of subproportional probability weights $w\left(s^{t}\right)$.

\section{Discussion}

It has long been recognized that uncertainty is an important dimension of choices over time. While we cannot claim credit for this longstanding idea, which goes back at least to Fisher (1930), we provide novel insights into the relationship between risk taking and time discounting behavior. We show that risk preferences may not only play a role in determining the magnitude of the discount rate, as many economists have asserted, but also in generating decreasing impatience. Specifically, our results suggest that people's proneness to nonlinear probability weighting is an important source of hyperbolic discounting patterns.

Our experimental findings provide support for the existence of such a link between probability weighting and time discounting. In particular, we present the first evidence that more pronounced systematic departures from linear probability weighting indeed 
predict more strongly declining impatience. This result is robust to inclusion of additional controls, econometric approach as well as model specification. In fact, the only variable explaining a substantial fraction of heterogeneity in individual discounting patterns turns out to be the degree of nonlinearity of probability weights.

Furthermore, our approach provides a powerful unifying framework for explaining previous puzzling findings in intertemporal choice. It provides a deeper understanding of why immediacy diminishes when risk comes into play. When explicitly manipulating the level of risk, Keren and Roelofsma (1995) found that subjects' preference for a smaller immediate payment dwindled drastically when both immediate and delayed options were made risky. This finding led them to tentatively conclude that immediacy is likely to be a derivative of certainty, an intuition that we incorporate into our approach in a principled way. Furthermore, intrinsic uncertainty in conjunction with nonlinear probability weighting offers an explanation for the sign effect, the delay dependence of risk tolerance as well as the observed subadditivity of discount rates. It also provides a rationale for the finding that subjective ratings of the probability of receiving a future reward decline hyperbolically with the length of delay.

If probability weighting plays such an important role in discounting behavior, the obvious question concerning the source of these probability distortions arises. A number of theoretical contributions invoke emotions to explain behavior (Wu, 1999; Caplin and Leahy, 2001). Walther (2003, 2007), for instance, rationalizes nonlinear probability weighting by generalizing expected utility theory: He assumes that, in addition to monetary outcomes, the decision maker cares about emotions triggered by the resolution of uncertainty. His approach predicts that, if the decision maker anticipates experiencing elation or disappointment when the actual outcome lies above or below some normal level, she will distort outcome probabilities according to an S-shaped pattern. The more emotional a person expects to be, the stronger will be her departure from linear probability weighting and, consequently, the larger her decline of discount rate. Of course, sensitivity to anticipated emotions is not easily observable, and we have to leave it to future research to investigate whether anticipated emotions are the primary drivers of probability weighting.

What are the implications of our results for economic policy? There has been a long debate concerning people's chronically low propensity to save, possible stemming from hyperbolic discounting. Our analysis suggests that hyperbolicity is caused by people's subjective assessment of future uncertainty. Uncertainty may stem from different sources, either tied to the individual herself, such as lifetime expectancy, or to environmental factors. Lack of contract enforcement and weak property rights, for instance, may make people skeptical that promises will be actually kept. Therefore, institutionally 
generated uncertainties may induce extreme short-run impatience even if people's pure rate of time preference is constant. In accordance with this view, discounting patterns in developing countries, the institutions of which are typically characterized by highly imperfect contract enforcement and weak property rights, seem to depart much more pronouncedly from exponentiality that in Western societies. Our approach suggest that policy measures and regulations fostering well defined property rights, reliable institutions of contract enforcement, trustworthy financial institutions, as well as general norms of pacta sunt servanda help on reducing excessive short-run impatience as well as overall high discount rates.

Aside from reducing uncertainty through institutional reforms, another channel to affect people's behavior could be their proneness to probability distortion. Unfortunately, little is known about the forces driving probability weighting, but we speculate that, aside from emotional factors, cognitive limitations as well as familiarity with the decision domain may play a decisive role here. If this is the case, policy measures should include education and information. All of these measures do not promise immediate success and, therefore, require a long term view on the part of the policy makers.

While our analysis helps to gain a better understanding of one of the mechanisms behind discounting behavior, this discussion makes clear that the reasons why people apparently distort probabilities are still not well understood and should, therefore, become an object of future research. 


\section{A Theoretical Framework}

In the following we derive hyperbolicity of discounting from our assumptions on intrinsic uncertainty and nonlinear probability weighting. Furthermore, we show that the extent of hyperbolicity is a consequence of the degree of subproportionality of the probability weighting function. Finally, we demonstrate that higher uncertainty induces greater hyperbolicity.

\section{A.1 Hyperbolicity}

In the framework proposed here, the discount factor $D(t)$ equals

$$
D(t)=\rho^{t} w\left(s^{t}\right)
$$

with $\rho$ defined as $\exp (-r)$. The discount function $D(t)$ is exponential if $w\left(s^{t}\right)=s^{t}$. Obviously, in this case, uncertainty per se reduces the discount factor by $s^{t}$, and hence increases the imputed per-period discount rate to $d=r-\ln (s)>r$ for $0<s<1$, but does not interfere with exponentiality of the discount function. If the probability of contract survival is weighted nonlinearly, however, the discount function will depart from exponentiality.

In order to establish the link between subproportional probability weighting and hyperbolicity, we define decreasing impatience at $t$ in the following way (Prelec, 1989, 2004): Let $(x, t)$ be a temporal prospect paying off $x$ at $t$ with certainty. A preference relation $\succeq$ exhibits decreasing impatience if for any $t>0,0<x<y,(x, u) \sim(y, z)$ implies $(y, z+t) \succeq(x, u+t)$.

According to our framework the temporal prospects $(x, 0) \sim(y, 1)$ are evaluated as $v(x) \rho^{0} w\left(s^{0}\right)=v(y) \rho^{1} w\left(s^{1}\right)$. As subproportionality of $w$ implies that $w(s)<w\left(s^{t+1}\right) / w\left(s^{t}\right)$, deferring the prospects by $t$ periods renders

$$
1=\frac{v(y) \rho w(s)}{v(x)}<\frac{v(y) \rho^{t+1} w\left(s^{t+1}\right)}{v(x) \rho^{t} w\left(s^{t}\right)}
$$

and, therefore, $(y, t+1) \succ(x, t)$, meeting the requirement for decreasing impatience.

In the intertemporal tradeoff between the present and the subsequent period the discount factor equals $\rho w(s)$. At time $t, v(x)$ is discounted by $\rho^{t} w\left(s^{t}\right)$. Compounding by the initial one-period discount factor $\rho w(s)$ would render $\rho^{t+1} w(s) w\left(s^{t}\right)$ at $t+1$, but the discount factor effectively amounts to $\rho^{t+1} w\left(s^{t+1}\right)$ then. Therefore, $w\left(s^{t+1}\right) /\left(w(s) w\left(s^{t}\right)\right)$, the wedge between the relative discount factors $D(0) / D(1)$ and $D(t) / D(t+1)$, provides a measure for the extent of departure from stationarity at $t$. 


\section{A.2 Effect of Concavity}

In the course of the experiment we cannot observe discount factors at delay $t, D(t)$, directly, however, but infer $\tilde{D}(t)$ from the intertemporal tradeoffs between payments at different dates, i.e. $y_{0}=\tilde{D}(t) y_{t}$. According to our assumptions, utility is modeled by a power function $v(y)=y^{\eta}, \eta>0$, which renders $\tilde{D}(t)=D(t)^{\frac{1}{\eta}}$. It follows that

$$
\frac{\tilde{D}(0) / \tilde{D}(1)}{\tilde{D}(t) / \tilde{D}(t+1)}=\left(\frac{D(0) / D(1)}{D(t) / D(t+1)}\right)^{\frac{1}{\eta}}
$$

and therefore observed decreasing impatience resulting from nonlinear probability weighting gets amplified by $\eta<1$ and, hence, concavity has to be controlled for in the regression model.

\section{A.3 Comparative Hyperbolicity}

According to our framework, decreasing impatience can solely be rationalized by nonlinear probability weighting. Consequently, a clear hypothesis results which can be tested empirically: Decision makers prone to probability weighting may be also prone to decreasing impatience. As we show below, we can derive a more precise prediction: A decision maker with a comparatively more subproportional probability weighting function will also tend to exhibit more decreasing impatience. This relationship provides the basis for our empirical test.

A preference relation $\succeq_{2}$ exhibits more decreasing impatience than $\succeq_{1}$ if for any intervals $0 \leq u<z, t, \Delta t$ and outcomes $0<x<y, 0<x^{\prime}<y^{\prime},(x, u) \sim_{1}(y, z)$, $(x, u+t) \sim_{1}(y, z+t+\Delta t)$, and $\left(x^{\prime}, u\right) \sim_{2}\left(y^{\prime}, z\right)$ imply $\left(x^{\prime}, u+t\right) \preceq_{2}\left(y^{\prime}, z+t+\Delta t\right)$ (Prelec, 2004).

In order to examine the effect of the degree of subproportionality on hyperbolicity suppose that the probability weighting function $w_{2}$ is comparatively more subproportional than $w_{1}$, as defined in Prelec (1998), and that the following indifference relations hold for two decision makers 1 and 2 at periods $u=0$ and $z=1$ :

$$
\begin{gathered}
v_{1}(x)=v_{1}(y) \rho w_{1}(s) \text { for } 0<x<y, \\
v_{2}\left(x^{\prime}\right)=v_{2}\left(y^{\prime}\right) \rho w_{2}(s) \text { for } 0<x^{\prime}<y^{\prime} .
\end{gathered}
$$

Due to subproportionality, in period $t$,

$$
1=\frac{v_{1}(y) \rho w_{1}(s)}{v_{1}(x)}<\frac{v_{1}(y) \rho^{t+1} w_{1}\left(s^{t+1}\right)}{v_{1}(x) \rho^{t} w_{1}\left(s^{t}\right)}
$$

Therefore, the subjective probability of contract survival has to be reduced by compound- 
ing $s$ over an additional time period $\Delta t$ to re-establish indifference:

$$
v_{1}(x) \rho^{t} w_{1}\left(s^{t}\right)=v_{1}(y) \rho^{t+1} w_{1}\left(s^{t+1+\Delta t}\right) .
$$

It follows from the definition of comparative subproportionality that this adjustment of the survival probability by $\Delta t$ is not sufficient to re-establish indifference with respect to $w_{2}$, i.e.

$$
v_{2}\left(x^{\prime}\right) \rho^{t} w_{2}\left(s^{t}\right)<v_{2}\left(y^{\prime}\right) \rho^{t+1} w_{2}\left(s^{t+1+\Delta t}\right) .
$$

Therefore, $\left(x^{\prime}, t\right) \prec\left(y^{\prime}, t+1+\Delta t\right)$. 


\section{A.4 Uncertainty and Hyperbolicity}

In order to derive the effect of increasing uncertainty on hyperbolicity we examine the sensitivity of the departure from stationarity at $t$, measured by $w\left(s^{t+1}\right) /\left(w(s) w\left(s^{t}\right)\right)$, with respect to changing $s$ :

$$
\begin{aligned}
& \frac{\partial}{\partial s}\left[\frac{w\left(s^{t+1}\right)}{w(s) w\left(s^{t}\right)}\right] \\
= & \frac{1}{\left[w(s) w\left(s^{t}\right)\right]^{2}}\left[(1+t) s^{t} w(s) w\left(s^{t}\right) w^{\prime}\left(s^{t+1}\right)-t s^{t-1} w(s) w\left(s^{t+1}\right) w^{\prime}\left(s^{t}\right)-w\left(s^{t}\right) w\left(s^{t+1}\right) w^{\prime}(s)\right] \\
= & \frac{1}{s\left[w(s) w\left(s^{t}\right)\right]^{2}}\left[(1+t) s^{t+1} w(s) w\left(s^{t}\right) w^{\prime}\left(s^{t+1}\right)-t s^{t} w(s) w\left(s^{t+1}\right) w^{\prime}\left(s^{t}\right)-s w\left(s^{t}\right) w\left(s^{t+1}\right) w^{\prime}(s)\right] \\
= & \frac{w\left(s^{t+1}\right)}{s w(s) w\left(s^{t}\right)}\left[\frac{(1+t) s^{t+1} w^{\prime}\left(s^{t+1}\right)}{w\left(s^{t+1}\right)}-\frac{t s^{t} w^{\prime}\left(s^{t}\right)}{w\left(s^{t}\right)}-\frac{s w^{\prime}(s)}{w(s)}\right] \\
= & \frac{w\left(s^{t+1}\right)}{s w(s) w\left(s^{t}\right)}\left[(1+t) \varepsilon\left(s^{t+1}\right)-t \varepsilon\left(s^{t}\right)-\varepsilon(s)\right] \\
< & 0
\end{aligned}
$$

with $\varepsilon(p)=p w^{\prime}(p) / w(p)$ defined as the elasticity of the probability weighting function $w$. According to Lemma 4.1 in Segal (1987), subproportionality holds iff $\varepsilon(p)$ is increasing. As $s^{t+1}<s^{t}<s, \varepsilon\left(s^{t+1}\right)<\varepsilon\left(s^{t}\right)<\varepsilon(s)$ and, hence, the sum of the elasticities in the final line of the derivation is negative. Therefore, increasing uncertainty, i.e. decreasing s, entails a greater departure from stationarity and, consequently, a higher degree of hyperbolicity. 


\section{B Censored Regression Model}

This appendix discusses the way we model the difference in the censored observed discount rates, $\Delta \delta=\delta_{02}-\delta_{04}$, and link it to individual risk preferences.

To relate time discounting to risk preferences, the model assumes the following linear relationship between the discount rate $\delta_{k, i}^{*}$ of individual $i \in\{1, \ldots, N\}$ over delay $k \in$ \{two months, four months\} and a vector of regressors $c_{i}$, containing a constant, the parameters of risk preferences, $\eta_{i}$ and $\alpha_{i}$, as well as some socioeconomic characteristics:

$$
\delta_{k, i}^{*}=c_{i} \beta_{k}+e_{k, i}
$$

where $\beta_{k}$ denotes a vector of slope parameters and $e_{k, i}$ stands for a normally distributed error term with mean zero and variance $\frac{1}{2} \sigma^{2}$. Under the assumption of nonnegative discounting, the choice menu, depicted in Figure III, allows us to directly elicit individual discount rates, which lie between $0 \%$ and $92.5 \%$. However, if individual $i$ always opts for being paid out at the earlier point in time (Option A), we do not necessarily observe her true discount rate $\delta_{k, i}^{*}$ as we only know that it amounts to at least $95 \%$. Thus, the elicited discount rates, $\delta_{02, i}$ and $\delta_{04, i}$, are censored from above at $b=0.95$. In the data we observe

$$
\delta_{k, i}= \begin{cases}\delta_{k, i}^{*} & \text { if } \delta_{k, i}^{*}<b \\ b & \text { otherwise }\end{cases}
$$

This immediately yields the difference in the discount rates over two and four months,

$$
\Delta \delta_{i}^{*}=c_{i} \underbrace{\left(\beta_{02}-\beta_{04}\right)}_{\Delta \beta}+\underbrace{e_{02, i}-e_{04, i}}_{\Delta e_{i}}
$$

where $\Delta e_{i}$ is normally distributed with mean zero and variance $\sigma^{2}$. Consequently, this difference $\Delta \delta_{i}^{*}$ is affected by censoring, too, and only observed if both $\delta_{02, i}<b$ and $\delta_{04, i}<b$.

In order to avoid biased estimators for $\beta_{02}, \beta_{04}$, and $\sigma$, the model needs to take the censored nature of the data into account. Therefore, its log likelihood takes on the 
following form:

$$
\begin{aligned}
\ln L\left(\beta_{02}, \beta_{04}, \sigma ; c, \delta_{02}, \delta_{04}\right) & =\sum_{i: \delta_{02, i}=b, \delta_{04, i}=b} P\left(\delta_{02, i}=b, \delta_{04, i}=b \mid c, \delta_{02}, \delta_{04}\right)(\mathrm{A} .10) \\
& +\sum_{i: \delta_{02, i}<b, \delta_{04, i}=b} P\left(\delta_{02, i}<b, \delta_{04, i}=b \mid c, \delta_{02}, \delta_{04}\right) \\
& +\sum_{i: \delta_{02, i}=b, \delta_{04, i}<b} P\left(\delta_{02, i}=b, \delta_{04, i}<b \mid c, \delta_{02}, \delta_{04}\right) \\
& +\sum_{i: \delta_{02, i}<b, \delta_{04, i}<b} \frac{1}{\sigma} \phi\left(\frac{\Delta \delta_{i}-c_{i}\left(\beta_{02}-\beta_{04}\right)}{\sigma}\right),
\end{aligned}
$$

where $\phi$ represents the standard normal distribution's density and the probabilities $P$, accounting for the different ways by which an observation may be censored, are given by

$$
\begin{aligned}
P\left(\delta_{02, i}=b, \delta_{04, i}=b \mid c, \delta_{02}, \delta_{04}\right) & =\left[1-\Phi\left(\frac{b-c_{i} \beta_{02}}{\sigma}\right)\right]\left[1-\Phi\left(\frac{b-c_{i} \beta_{04}}{\sigma}\right)\right], \\
P\left(\delta_{02, i}<b, \delta_{04, i}=b \mid c, \delta_{02}, \delta_{04}\right) & =\Phi\left(\frac{b-c_{i} \beta_{02}}{\sigma}\right)\left[1-\Phi\left(\frac{b-c_{i} \beta_{04}}{\sigma}\right)\right], \\
P\left(\delta_{02, i}=b, \delta_{04, i}<b \mid c, \delta_{02}, \delta_{04}\right) & =\left[1-\Phi\left(\frac{b-c_{i} \beta_{02}}{\sigma}\right)\right] \Phi\left(\frac{b-c_{i} \beta_{04}}{\sigma}\right),
\end{aligned}
$$

with $\Phi$ denoting the cumulative density function of the standard normal distribution. Numerical maximization of $\ln L\left(\beta_{02}, \beta_{04}, \sigma ; c, \delta_{02}, \delta_{04}\right)$ yields the maximum likelihood estimates of $\hat{\beta}_{02}, \hat{\beta}_{04}$, and $\hat{\sigma}$. To obtain the maximum likelihood estimate of $\Delta \hat{\beta}$ we utilize the invariance property. 


\section{Observed Discount Rates}

Figure A.1: Discount Rates over 2 and 4 Months, $\delta_{02}$ and $\delta_{04}$
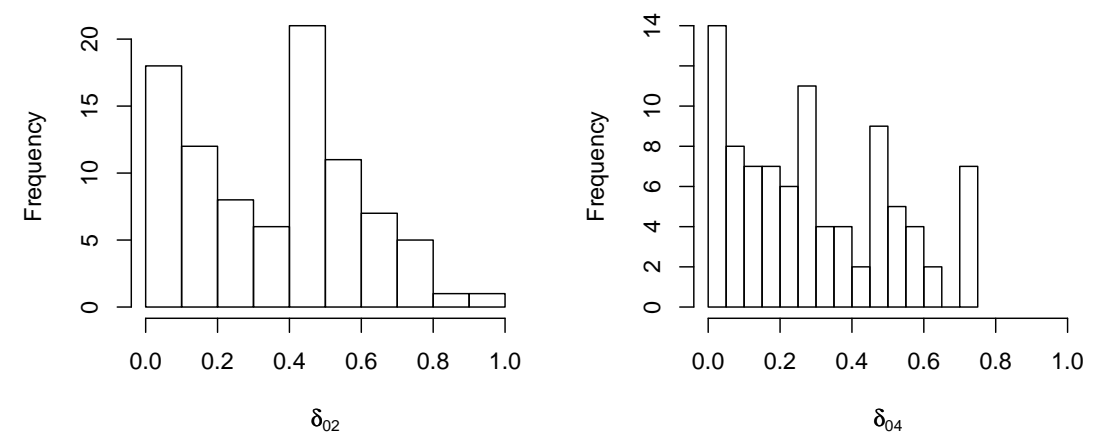

Figure A.2: Change in Discount Rates $\Delta \delta$

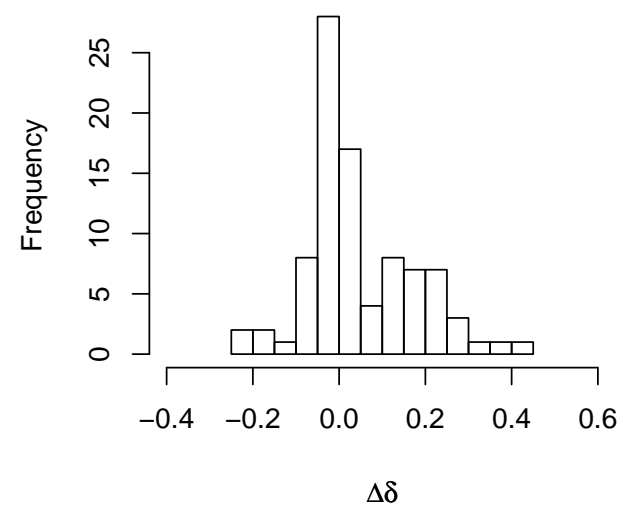




\section{Estimated Risk Parameters}

Figure A.3: Distribution of $\eta$ and $\alpha$
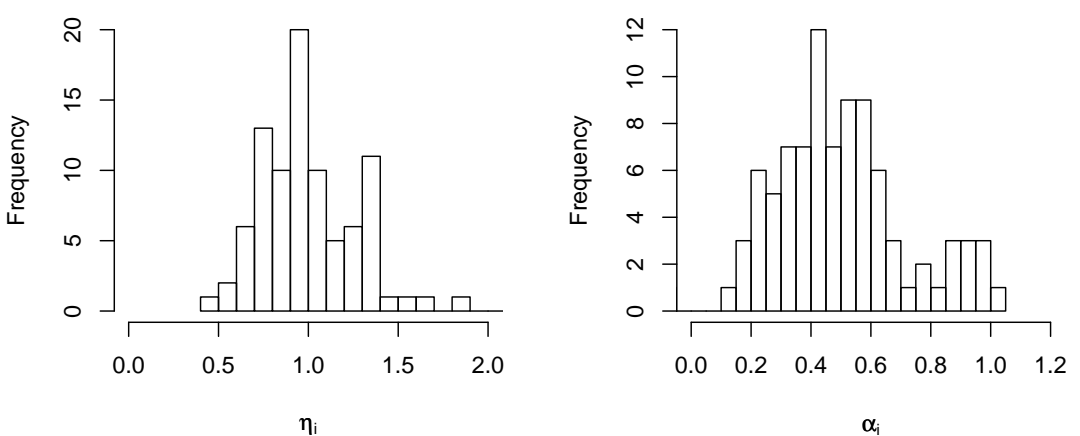


\section{E Controls}

Table A.1: Summary Statistics $(n=114)$

\begin{tabular}{rlrr}
\hline \hline & Type & Mean & Std.Err. \\
\hline Female & binary & 0.439 & 0.047 \\
Age & numeric & 22.640 & 0.206 \\
Log-Income & numeric & 6.388 & 0.066 \\
Experience & binary & 0.307 & 0.043 \\
CRT & numeric & 2.202 & 0.081 \\
\hline \hline
\end{tabular}

Table A.2: Number of Observations at the Bounds $(n=114)$

\begin{tabular}{rrrr}
\hline \hline & $\delta_{02}$ & $\delta_{04}$ & $\delta_{24}$ \\
\hline$\geq 95 \%$ & 24 & 15 & 16 \\
$0 \%$ & 2 & 0 & 3 \\
\hline \hline
\end{tabular}




\section{F Alternative Specification}

Alternatively to Prelec's specification of the probability weighting function we also estimated the risk model based on Karmarkar's proposal (Karmarkar, 1979):

$$
w(p)=\frac{p^{\gamma}}{p^{\gamma}+(1-p)^{\gamma}}, 0<\gamma
$$

This function is inverted S-shaped for $0<\gamma<1$, subproportional for medium and large probabilities. It intersects the diagonal form above at $p=0.5$. The parameter $\gamma$ measures departure from linear probability weighting. The larger is $\gamma$, the less strongly S-shaped is the curve. As Table A.3 shows, the relationship between hyperbolic discounting and nonlinear probability weighting is equally strong and significant as for Prelec's specification.

Table A.3: Karmarkar Specification

\begin{tabular}{|c|c|c|}
\hline \multicolumn{3}{|c|}{ Dependent Variable: $\Delta \delta\left(\Delta \delta^{*}\right)$} \\
\hline & $\overline{\text { OLS }}^{a}$ & Censored \\
\hline \multirow[t]{2}{*}{ Intercept } & 0.161 & 0.147 \\
\hline & $(0.051)$ & $(0.047)$ \\
\hline \multirow[t]{2}{*}{$\eta$} & 0.016 & 0.027 \\
\hline & $(0.051)$ & $(0.048)$ \\
\hline \multirow[t]{2}{*}{$\gamma$} & $-0.212^{* *}$ & $-0.186^{* *}$ \\
\hline & $(0.068)$ & $(0.062)$ \\
\hline$\hat{\sigma}$ & 0.123 & 0.085 \\
\hline$R^{2}$ or (LogLik) & 0.121 & $(45.029)$ \\
\hline Observations & 90 & 114 \\
\hline Parameters & 3 & 7 \\
\hline \multicolumn{3}{|c|}{$\begin{array}{l}\text { a) without censored observations. }{ }^{* *} \text { significant } \\
\text { at the } 1 \% \text { level. Bootstrapped standard errors } \\
\text { in parentheses }(10,000 \text { replications). Bootstrap- } \\
\text { ping accounts for the fact that } \gamma \text { and } \eta \text { are } \\
\text { estimated quantities. }\end{array}$} \\
\hline
\end{tabular}




\section{References}

Abdellaoui, M. (2000): "Parameter-Free Elicitation of Utility and Probability Weighting Functions," Management Science, 46(11), 1497-1512.

Ainslie, G. (1992): "Picoeconomics: The Interaction of Successive Motivational States Within the Person," Cambridge University Press.

Anderson, C. L., M. Dietz, A. Gordon, and M. Klawitter (2004): "Discount Rates in Vietnam," Economic Development and Cultural Change, 52(4), 873-887.

Angeletos, G., D. Laibson, A. Repetto, and J. Tobacman (2001): "The Hyperbolic Consumption Model: Calibration, Simulation, and Empirical Evaluation," Journal of Economic Perspectives, 15(3), 47-68.

Benzion, U., A. Rapoport, And J. Yagil (1989): "Discount Rates Inferred from Decisions: An Experimental Study," Management Science, 35(3), 270-284.

Bruhin, A., H. Fehr-Duda, and T. Epper (2007): "Risk and Rationality: Uncovering Heterogeneity in Probability Distortion," SOI Working Paper Series, University of Zurich (Switzerland), 0705.

Bruhin, A., H. Fehr-Duda, and T. Epper (2008): "Risk and Rationality: Uncovering Heterogeneity in Probability Distortion," SOI Working Paper Series, 0705.

Caplin, A., And J. Leahy (2001): "Psychological Expected Utility Theory and Anticipatory Feelings," Quarterly Journal of Economics, 116(1), 55-79.

Chapman, G. (1996): "Temporal Discounting and Utility for Health and Money," Journal of Experimental Psychology, 22(3), 771-791.

Chapman, G., And A. S. Elstein (1995): "Valuing the Future: Temporal Discounting of Health and Money," Medical Decision Making, 15(4), 373-386.

Coller, M., and M. Williams (1999): "Eliciting Individual Discount Rates," Experimental Economics, 2, 107-127.

Dohmen, T., A. Falk, D. Huffman, and U. Sunde (2007): "Dynamic Inconsistency is Pervasive among Humans and Predicts Self-Control Problems," Institute for the Study of Labor (IZA), Bonn (Germany), mimeo.

Fehr-Duda, H., M. De Gennaro, and R. Schubert (2006): "Gender, Financial Risk, and Probability Weights," Theory and Decision, 60, 283-313. 
Fischbacher, U. (2007): "z-Tree: Zurich Toolbox for Ready-Made Economic Experiments," Experimental Economics, 10, 171-178.

FISHER, I. (1930): "The Theory of Interest as Determined by Impatience to Spend Income and Opportunity to Invest it," New York: Macmillan.

Frederick, S. (2005): "Cognitive Reflection and Decision Making," Journal of Economic Perspectives, 19(4), 25-42.

Frederick, S., G. Loewenstein, and T. O'Donoghue (2002): "Time Discounting and Time Preference: A Critical Review," Journal of Economic Literature, 40, 351-401.

FudenberG, D., And D. K. Levine (2009): "Self Control, Risk Aversion, and the Allais Paradox," mimeo.

Gonzalez, R., And G. Wu (1999): "On the Shape of the Probability Weighting Function," Cognitive Psychology, 38, 129-166.

Halevy, Y. (2008): "Strotz Meets Allais: Diminishing Impatience and the Certainty Effect," American Economic Review, 98(3), 1145-1162.

Hey, J. D., And L. Jinkwon (2005): "Do Subjects Separate (or Are They Sophisticated)?," Experimental Economics, 8, 233-265.

Kahneman, D., And A. Tversky (1979): "Prospect Theory: An Analysis of Decision under Risk," Econometrica, 47(2), 263-291.

Karmarkar, U. S. (1979): "Subjectively Weighted Utility and the Allais Paradox," Organizational Behavior and Human Performance, 24, 67-72.

KarP, L. (2005): "Global Warming and Hyperbolic Discounting," Journal of Public Economics, 89, 261-282.

Keren, G., and P. Roelofsma (1995): "Immediacy and Certainty in Intertemporal Choice," Organizational Behavior and Human Decision Processes, 63(3), 287-297.

KIRBY, K. (1997): "Bidding on the Future: Evidence Against Normative Discounting of Delayed Rewards," Journal of Experimental Psychology, 126(1), 54-70.

Kirby, K., and N. Maraković (1995): "Modeling Myopic Decisions: Evidence for Hyperbolic Delay-Discounting within Subjects and Amounts," Organizational Behavior and Human Decision Processes, 64(1), 22-30. 
Laibson, D. (1997): "Golden Eggs and Hyperbolic Discounting," Quarterly Journal of Economics, 112(2), 443-477.

(1998): "Life-Cycle Consumption and Hyperbolic Discount Functions," European Economic Review, 42, 861-871.

Laibson, D., A. Repetto, J. Tobacman, R. Hall, W. Gale, and G. Akerlof (1998): "Self-Control and Saving for Retirement," Brookings Papers on Economic Activity, 1998(1), 91-196.

Lattimore, P., J. Baker, and A. D. Witte (1992): "The Influence of Probability on Risky Choice: A Parametric Examination," Journal of Economic Behavior and Organization, 17, 377-400.

Loewenstein, G., And D. Prelec (1992): "Anomalies in Intertemporal Choice: Evidence and an Interpretation," Quarterly Journal of Economics, 107(2), 573-597.

Myerson, J., And L. Green (1995): "Discounting of Delayed Rewards: Models of Individual Choice," Journal of the Experimental Analysis of Behavior, 64, 263-276.

Noussair, C., And P. Wu (2006): "Risk Tolerance in the Present and the Future: An Experimental Study," Managerial and Decision Economics, 27, 401-412.

O’Donoghue, T., And M. Rabin (1999): "Doing It Now or Later," American Economic Review, 89(1), 103-124.

(2001): "Choice and Procrastination," Quarterly Journal of Economics, 116(1), $121-160$.

Patak, M., and B. Reynolds (2007): "Question-based Assessment of Delay Discounting: Do Respondents Spontaneously Incorporate Uncertainty into their Valuations for Delayed Rewards?," Addictive Behaviors, 32, 351-357.

Poulos, C., And D. Whittington (2000): "Time Preferences for Life-Saving Programs: Evidence from Six Less Developed Countries," Environmental Science and Technology, 34(8), 1445-1455.

Prelec, D. (1989): "Decreasing Impatience: Definition and Consequences," Harvard Business School Working Paper, (90-015).

(1998): "The Probability Weighting Function," Econometrica, 66(3), 497-527. 
(2004): "Decreasing Impatience: A Criterion for Non-stationary Time Preference and 'Hyperbolic' Discounting," The Scandinavian Journal of Economics, 106(3), 511-532.

Prelec, D., and G. Loewenstein (1991): "Decision Making Over Time and Under Uncertainty: A Common Approach," Management Science, 37(7), 770-786.

Rachlin, H., A. Raineri, And D. Cross (1991): "Subjective Probability and Delay," Journal of the Experimental Analysis of Behavior, 55(2), 233-244.

READ, D. (2001): "Is Time-Discounting Hyperbolic or Subadditive?," Journal of Risk and Uncertainty, 23(1), 5-32.

Richards, J., L. Zhang, S. Mitchell, and H. D. Wit (1999): "Delay or Probability Discounting in a Model of Impulsive Behavior: Effect of Alcohol," Journal of the Experimental Analysis of Behavior, 71(2), 121-143.

Segal, U. (1987): "The Ellsberg Paradox and Risk Aversion: An Anticipated Utility Approach," International Economic Review, 28(1), 175-202.

Starmer, C., And R. Sugden (1989): "Violations of the Independence Axiom in Common Ratio Problems: An Experimental Test of Some Competing Hypotheses," Annals of Operations Research, 19, 79-102.

Sтотт, H. (2006): "Cumulative Prospect Theory's Functional Menagerie," Journal of Risk and Uncertainty, 32, 101-130.

Takahashi, T., K. Ikeda, T. Hasegawa, and A. Greene (2007): "A hyperbolic decay of subjective probability of obtaining delayed rewards," Behavioral and Brain Functions, 52(3).

Thaler, R. (1981): "Some Empirical Evidence on Dynamic Inconsistency," Economics Letters, 8, 201-207.

Tversky, A., And D. Kahneman (1992): "Advances in Prospect Theory: Cumulative Representation of Uncertainty," Journal of Risk and Uncertainty, 5, 297-323.

Walther, H. (2003): "Normal-Randomness Expected Utility, Time Preference and Emotional Distortions," Journal of Economic Behavior and Organization, 52, 253-266.

(2007): "S-shaped Probability Weighting and Hyperbolic Preference Reversal - An Intimate Relationship," Vienna University of Economics and Business Administration (Austria), mimeo. 
Wu, G. (1999): "Anxiety and Decision Making with Delayed Resolution of Uncertainty," Theory and Decision, 46, 159-198. 